\title{
ARTICLE
}

Received 28 May 2013 | Accepted 22 Oct 2013 | Published 15 Nov $2013 \quad$ DOl: 10.1038/ncomms3804

\section{Solid-source growth and atomic-scale characterization of graphene on $\mathrm{Ag}(111)$}

Brian Kiraly ${ }^{1,2, \star}$, Erin V. Iski ${ }^{1, \star}$, Andrew J. Mannix ${ }^{1,2}$, Brandon L. Fisher ${ }^{1}$, Mark C. Hersam ${ }^{2,3}$ \& Nathan P. Guisinger ${ }^{1}$

Silver is a desirable platform for graphene growth because of the potential for hybrid graphene plasmonics and its emerging role as a preferred growth substrate for other twodimensional materials, such as silicene. Here we demonstrate the direct growth of monolayer graphene on a single-crystal $\mathrm{Ag}(111)$ substrate. The inert nature of $\mathrm{Ag}$ has made it difficult to use for graphene synthesis using standard chemical vapour deposition techniques, which we have overcome by using an elemental carbon source. Atomic-scale scanning tunnelling microscopy reveals that the atomically clean graphene-silver substrate is free of organic residue and other contaminants. The dendritic graphene possesses a variety of edge terminations, many of which give rise to quantum interferences previously seen only on insulating substrates. This scattering supports spectroscopic evidence that the graphene electronic structure is minimally perturbed by the underlying silver, providing a new system in which graphene is decoupled from its growth substrate.

\footnotetext{
${ }^{1}$ Center for Nanoscale Materials, Argonne National Laboratory, 9700 South Cass Avenue, Building 440, Argonne, Illinois 60439, USA. ${ }^{2}$ Department of Materials Science and Engineering, Northwestern University, 2220 Campus Drive, Evanston, Illinois 60208, USA. ${ }^{3}$ Department of Chemistry, Northwestern University, 2220 Campus Drive, Evanston, Illinois 60208, USA. * These authors contributed equally to this work. Correspondence and requests for materials should be addressed to N.P.G. (email: nguisinger@anl.gov).
} 
G raphene exhibits well-known mechanical, thermal, electronic and optical properties that distinguish it from nearly all three-dimensional (3D) materials, thus motivating significant research in recent years ${ }^{1}$. To date, this research has primarily focused on the rich physics underlying graphene's properties; however, current attention has shifted to interfacing graphene with alternate materials, many of which are also twodimensional (2D), for new physics and device applications ${ }^{2-4}$. For example, hexagonal boron nitride substrates enable significant enhancements in electron mobility for graphene electronics ${ }^{5}$. Alternatively, integrated graphene plasmonics based on graphene interfaces with noble metals have realized architectures capable of extreme light concentration and manipulation, highly efficient photo-

conversion and single-molecule detection ${ }^{6,7}$. However, these interfaces are often prepared with and studied after significant material processing that contaminates the interface with a wide range of organic/inorganic species, thus compromising graphene's intrinsic characteristics ${ }^{8,9}$.

The direct growth of graphene at the desired interface provides a means to circumvent these issues and enables the fabrication of clean, well-defined interfaces. Furthermore, experiments conducted under ultra-high vacuum (UHV) permit the pristine and atomic-scale characterization of these systems, which enables elucidation of their intrinsic properties ${ }^{10}$. Graphene on $\mathrm{Ag}$ is an attractive system for these studies because of its excellent plasmonic characteristics and common use in printed electronics ${ }^{11,12}$. Even more compelling is the fact that $\mathrm{Ag}$ substrates have become a critical platform for novel 2D materials such as silicene, the silicon-based analogue to graphene, which has been prepared in UHV almost exclusively on $\operatorname{Ag}(111)^{13,14}$.

Here we report the growth of monolayer graphene on atomically pristine single-crystal $\mathrm{Ag}(111)$. Growth is accomplished by evaporating atomic carbon onto a $\mathrm{Ag}(111)$ surface at elevated temperatures under UHV conditions. The resulting graphene is examined at the atomic scale in situ via scanning tunnelling microscopy (STM) and scanning tunnelling spectroscopy (STS), and then further characterized via ex-situ Raman spectroscopy, scanning electron microscopy and X-ray photoelectron spectroscopy (XPS). Growth occurs at relatively low temperature compared with conventional chemical vapour deposition (CVD) techniques ${ }^{15-17}$, which suggests opportunities for integrating graphene with other low-melting-point substrates. The resulting graphene demonstrates electronic independence from the Ag substrate, as seen in STS. Furthermore, the mixed graphene edges give rise to quantum interferences, which are confined to the $\mathrm{C}-\mathrm{C}$ bonds, further corroborating the minimal effect of the Ag substrate on the electronic structure of the graphene ${ }^{18,19}$.

\section{Results}

Graphene growth morphology. Figure la shows a schematic representation of the growth process, depicting the carbon evaporation, surface diffusion and initial graphene growth. The atomic carbon is deposited from a graphite rod in a manner similar to molecular beam epitaxy, where the deposition rates are estimated to be on the order of $0.1 \mathrm{ML} \mathrm{min}^{-1}$. Figure $1 \mathrm{~b}$ shows a $500 \mathrm{~nm} \times 500 \mathrm{~nm}$ STM image of the $\mathrm{Ag}(111)$ surface after carbon deposition and graphene growth. The dendritic features emanating from the step edges and seen on the terraces are confirmed via atomic-resolution imaging (Fig. 1c) and STS measurements as graphene. Ex-situ scanning electron microscopy (Fig. 1d-h) can also be used to image the graphene coverage on the sample because of contrast arising from environmental degradation of the $\mathrm{Ag}(111)$ surface that reduces its electrical conductivity. Graphene (darker regions) is shown at full monolayer coverage in Fig. $1 \mathrm{~h}$. a

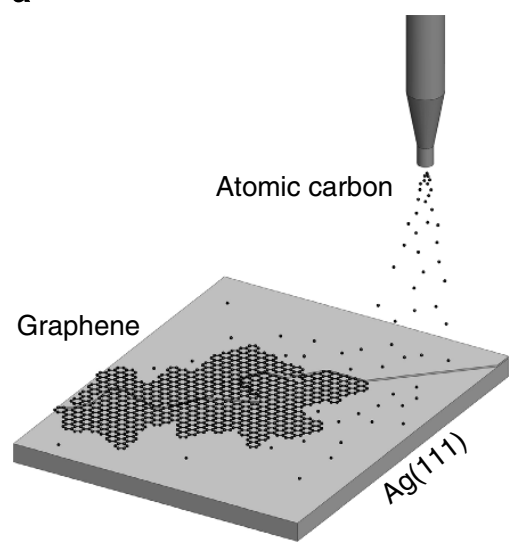

b

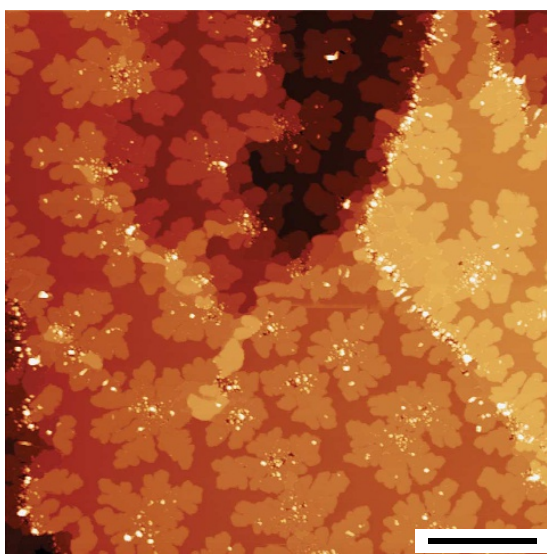

c

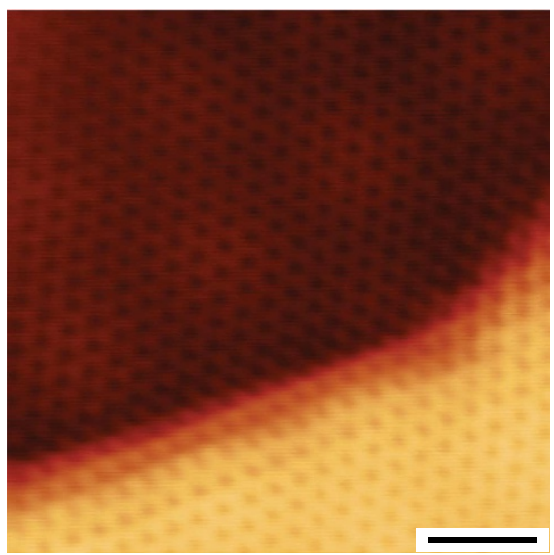

d

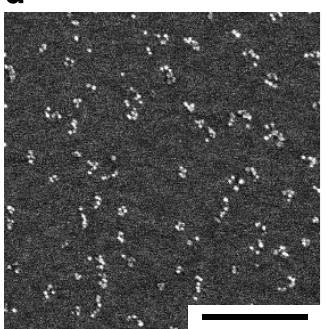

e

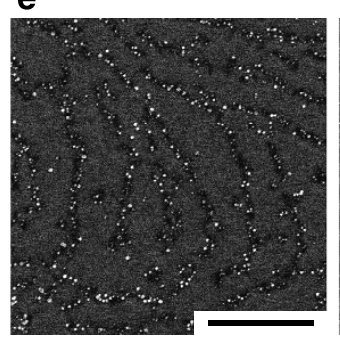

f

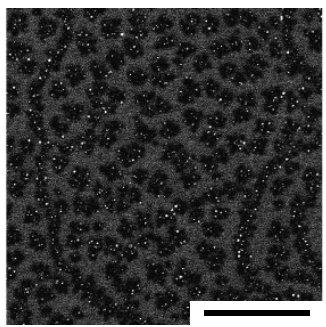

g

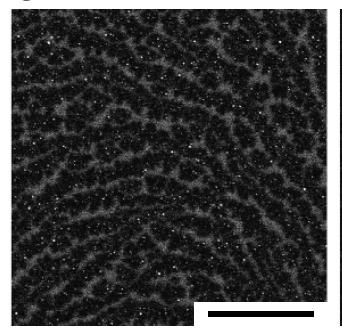

h

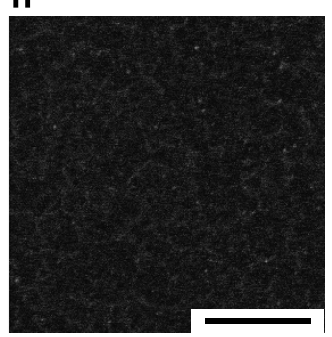

Figure 1 | Graphene on Ag(111). (a) Schematic of the growth process. (b) STM image of the resulting graphene morphology (scale bar, $100 \mathrm{~nm}, \mathrm{~V}=1 \mathrm{~V}$, $I=1 \mathrm{nA}$ ). (c) Atomic-resolution image of the graphene lattice across a Ag step (scale bar, $1 \mathrm{~nm}, V=25 \mathrm{mV}, I=15 \mathrm{nA}$ ). (d) Scanning electron microscopy (SEM) images after growth taken in regions with increasing carbon concentration from (d-h) with a full monolayer shown in $\mathbf{h}$ (scale bar, $500 \mathrm{~nm}$ in all SEM images). 
It is well known that low $\mathrm{C}$ solubility in $\mathrm{Cu}$ is an indicator of weak atomic interactions in the $\mathrm{Cu}-\mathrm{C}$ system; therefore, graphene growth on $\mathrm{Cu}$ has been understood and modelled by the diffusion of $\mathrm{C}$ species on $\mathrm{Cu}$ surfaces ${ }^{20,21}$. The comparably low solubility of $\mathrm{C}$ in $\mathrm{Ag}^{22}$ suggests that graphene growth on $\mathrm{Ag}(111)$ should follow a similar mechanism. The dendritic graphene growth is accompanied by small nanoparticles $(<10 \mathrm{~nm})$ of varying density and morphology on the $\operatorname{Ag}(111)$ surface as seen in Fig. 1b,d. The progression in Fig. $1 \mathrm{~d}-\mathrm{h}$ is obtained after growth at several points on the surface along a gradient in the carbon concentration, which resulted from the line-of-sight deposition. The graphene coverage increases with carbon concentration, as seen in Fig. 1d-h. The clusters that accompany graphene growth are difficult to characterize electronically because of their relatively high aspect ratios; however, atomically resolved images suggest that the graphene lattice extends over the top of the clusters (Supplementary Fig. S1). The observed graphene lattice, in addition to the centralized location of the clusters within the graphene domains, suggests that the clusters could be the nucleation points for graphene growth. Furthermore, the observation of similar clustering on $\mathrm{Cu}(111)$ (Supplementary Fig. S1e,f) indicates that the clusters are likely to be carbon-based and the growth behaviour is characteristic of the deposition method. Graphene nucleation after coalescence of carbon clusters has been discussed for graphene growth on $\mathrm{Ru}$ substrates ${ }^{23}$ and observed on $\mathrm{Rh}(111)^{24}$; however, the clusters are generally much smaller than the ones observed here. Furthermore, the cluster morphology exhibits significant temperature dependence, which has been used to reduce their density via post-growth annealing (Supplementary Fig. S2). Regardless of the exact nature of the nucleation, graphene growth is observed to originate both at atomic steps and on atomically flat terraces.

Figure $2 \mathrm{a}$ depicts the growth of graphene on an atomically flat terrace, in which graphene apparently grows outwards from a central particle(s)/nucleation point in a dendritic fashion. In general, the shape of the growth front is both temperature and carbon flux dependent, but demonstrates no repeated symmetry. In a different growth mode, graphene is observed to nucleate at the Ag step edges, shown in detail in Fig. $2 \mathrm{~b}$ and highlighted with dashed lines in Fig. 2d. Graphene growing from the Ag step edges generally causes significant deformation of the original Ag step. Although the cause of this deformation is difficult to isolate, it is plausible that the graphene restructured the steps during growth similar to etching or restructuring of metal steps during graphene growth on $\mathrm{Au}$ and $\mathrm{Rh}$, although this etching generally results in graphene terminating at the metal steps ${ }^{25,26}$. A linear topography profile across the graphene-covered step in Fig. 2b illustrates two major discontinuous features: a step at the interface between graphene and Ag (labelled b) and a step from one atomic terrace to another on the Ag crystal (labelled a). Although a matches well with expectations, $b$ is somewhat smaller $(\sim 2 \AA)$ than the expected $3.3 \AA$ at equilibrium ${ }^{27}$; however, it is difficult to draw conclusions from this fact, because this height can be affected by differences in tunnelling conductance between the two materials (seen in the STM topography data in Supplementary Fig. S3a-d). Furthermore, graphene is also observed to grow continuously across Ag step edges (Fig. 1c).

All graphene presented in this work was electronically confirmed with STS as a single layer. Although raised regions of growth appear at some terrace nucleation sites (indicated in Fig. 2d), STS data (inset Fig. 2d) demonstrate the electrical homogeneity of the upper and lower graphene (light blue), and its stark contrast to Ag (purple). Noting that the height difference between the two graphene regions is approximately the measured height of a Ag step, it appears that the raised graphene is growing on top of a Ag island on the terrace. Similar islands were observed
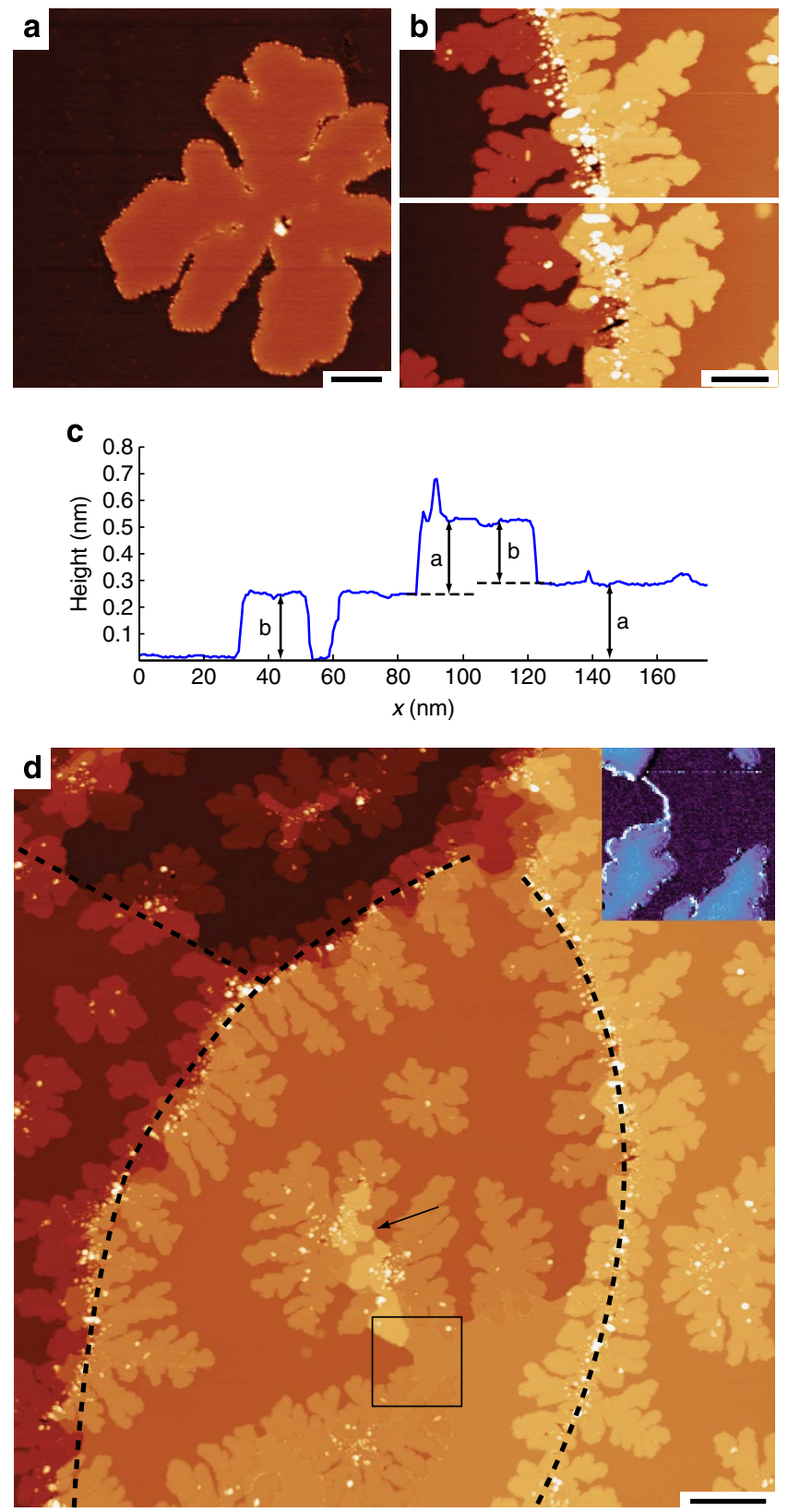

Figure 2 | Graphene growth morphology. (a) Growth of a graphene flake on an atomically flat terrace (scale bar, $10 \mathrm{~nm}, V=-600 \mathrm{mV}, I=2 \mathrm{nA}$ ). (b) Bidirectional growth of graphene from a Ag step edge (scale bar, $15 \mathrm{~nm}$, $V=1 \mathrm{~V}, I=1 \mathrm{nA}$ ). (c) Linear topography profile along the white line in b, with the graphene-Ag step labelled b and the Ag step labelled a. (d) Characteristic STM image of the growth morphology illustrating step and terrace nucleation (scale bar, $50 \mathrm{~nm}, V=1 \mathrm{~V}, I=1 \mathrm{nA}$ ). Boxed region (topography) and inset $(d l / d V)$ show a region where $\mathrm{Ag}$ atoms arrange at the edge of graphene growth with graphene appearing bright in the inset. The arrow indicates a region of graphene growing on a $\mathrm{Ag}$ island on the terrace.

to border graphene edges (see Fig. 1b) and growth on top of these islands would explain the electronic signature of monolayer graphene. The presence of monolayer graphene is also suggested by the symmetric $2 \mathrm{D}$ band (full width at half maximum $\left.(\mathrm{FWHM})=42 \mathrm{~cm}^{-1}\right)$ in the Raman $\mathrm{data}^{28}$. As seen in other studies, the growth of graphene from atomic carbon is not selflimiting $29-31$, with large one-dimensional objects appearing at higher coverages (Supplementary Fig. S4c). Supplementary Fig. S4b 
illustrates that these features are the result of colliding growth fronts of graphene flakes, which grow vertically (off of the surface) after meeting. These large features are likely to account for much of the roughness and disorder often seen in atomic source growth $^{31}$.

Ex-situ spectroscopy. The Raman spectrum in Fig. 3a shows the commonly observed $D\left(\sim 1,350 \mathrm{~cm}^{-1}\right), G\left(\sim 1,589 \mathrm{~cm}^{-1}\right)$ and 2D $\left(\sim 2,690 \mathrm{~cm}^{-1}\right)$ peaks for graphene ${ }^{32}$. In addition, the spectrum includes peaks at 1,625 and $2,940 \mathrm{~cm}^{-1}$, which are attributed to the defect-related $D^{\prime}$ band and the $D+G$ band, respectively ${ }^{28}$. The prominent $D$ band and the appearance of the $D^{\prime}$ and $D+G$ bands are generally related to point defects in the basal plane or edges of single-crystalline graphene domains ${ }^{33}$. We use two metrics from recent studies to suggest that the defectrelated peaks observed in Fig. $3 \mathrm{~d}$ are related to the high density of graphene boundaries in the observed growth process. First, the FWHM of the $D$ band is $\sim 25 \mathrm{~cm}^{-1}$. A study on graphene nanoribbons (GNRs) has shown that the $I(D) / I(G)$ ratio scales with the GNR width, but that the FWHM of the $D$ band, is constant and generally $<30 \mathrm{~cm}^{-1}$ if the basal plane of the GNR is unperturbed ${ }^{34}$. Second, the $I(D) / I\left(D^{\prime}\right)$ ratio of $\sim 4$ has been linked to a higher concentration of edge defects as opposed to vacancies or $s p^{3}$ carbon in the basal plane $e^{35}$. Noting that the laser spot size is $\sim 2-3 \mu \mathrm{m}$ (Fig. $2 \mathrm{~d}$ is $500 \mathrm{~nm} \times 500 \mathrm{~nm}$ or approximately an order of magnitude smaller in area), it is clear that the proportion of edges is much larger than graphene grown by CVD methods on $\mathrm{Cu}^{36}$. Furthermore, common STM observation of standing waves at mixed/armchair edges in the dendritic graphene indicates that a large portion of the edges are
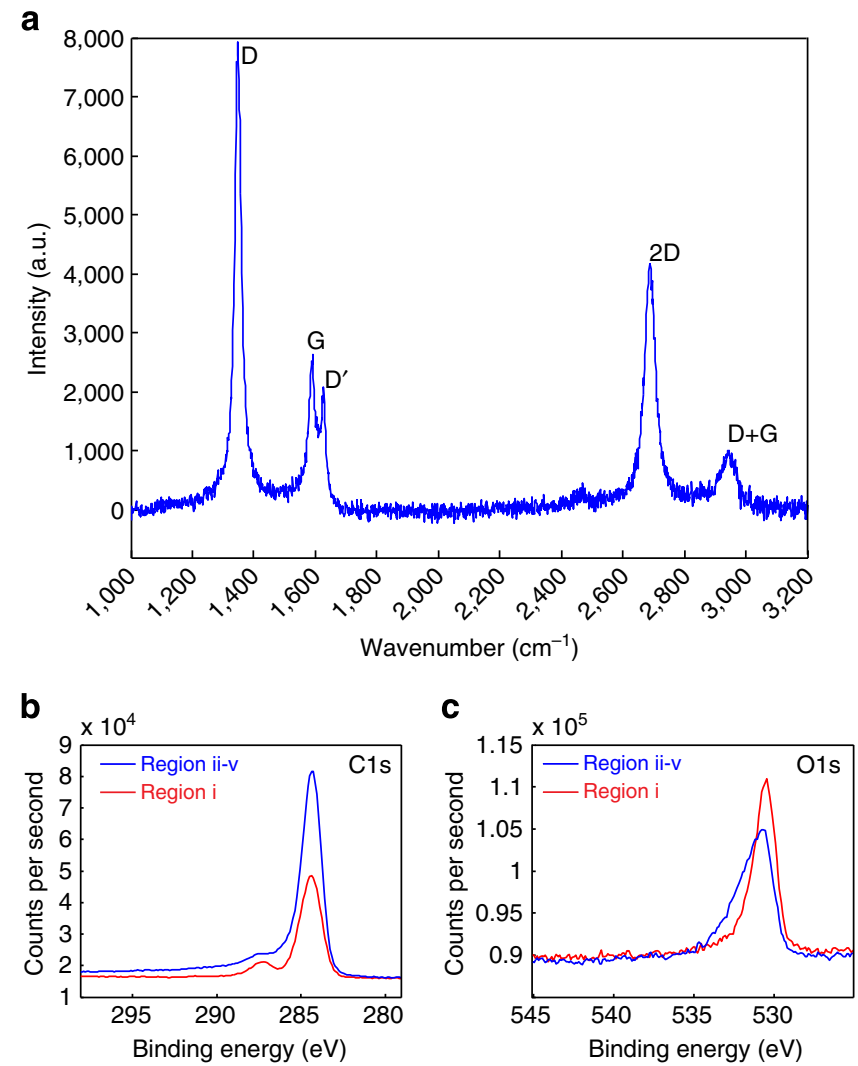

Figure 3 | Large-scale ex-situ characterization of graphene. (a) Raman spectrum of graphene on $\mathrm{Ag}(111)$. (b) C1s XPS spectrum from region $\mathrm{i}$ without graphene and region ii-v with graphene. (c) O1s XPS spectrum from regions $\mathrm{i}$ and $\mathrm{ii}-\mathrm{v}$. capable of supporting the intervalley scattering process associated with the presence of the Raman $D$ band ${ }^{37}$. The STM images also corroborate the lack of point defects on the basal plane, which are easily spotted because of their strong electronic scattering signatures $^{38}$.

Ex-situ XPS was used to further characterize the graphene-Ag system. Although the XPS sampling area was not small enough to distinguish Fig. 1e-h, it was possible to isolate regions devoid of graphene (Fig. 1d) from areas containing graphene. Figure $3 \mathrm{~b}$ shows the C1s spectra in regions $\mathbf{i}$ (Fig. 1d) and ii-v (Fig. 1e-h). It is clear that the $284.5 \mathrm{eV}$ peak intensity is significantly increased from region $\mathbf{i}$ to region $\mathbf{i i - v}$, indicating that the regions $\mathbf{i i - v}$ have
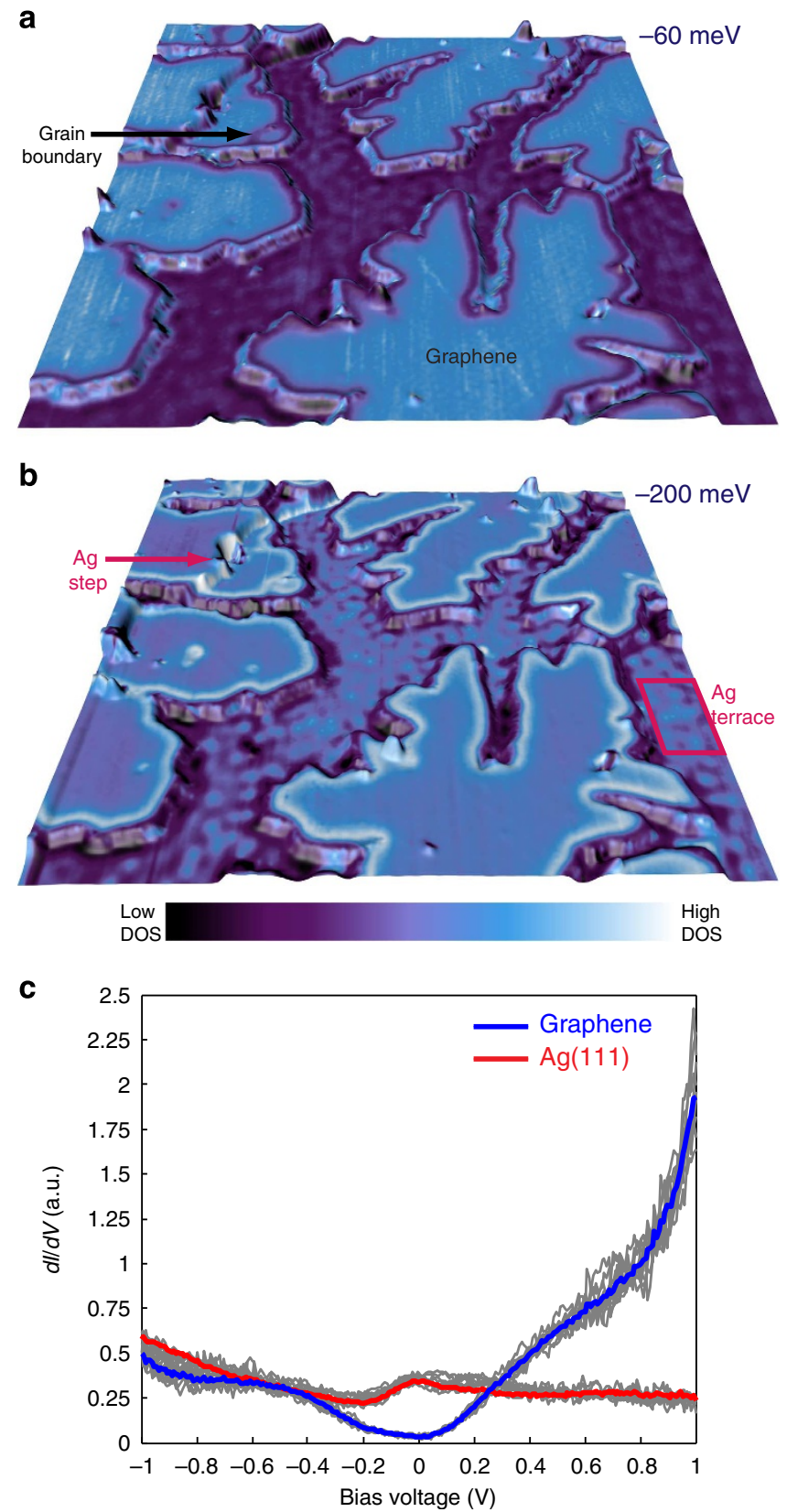

Figure 4 | Electronic characterization of graphene. (a) STS image collected at -200 meV superimposed on a $3 D$ rendering of the underlying topography. (b) STS image collected at $-60 \mathrm{meV}$ of the same region pictured in a superimposed on a 3D rendering of the underlying topography. Both images, $150 \mathrm{~nm} \times 150 \mathrm{~nm}$. (c) $d l / d V$ point spectra for graphene and pristine Ag. 
a
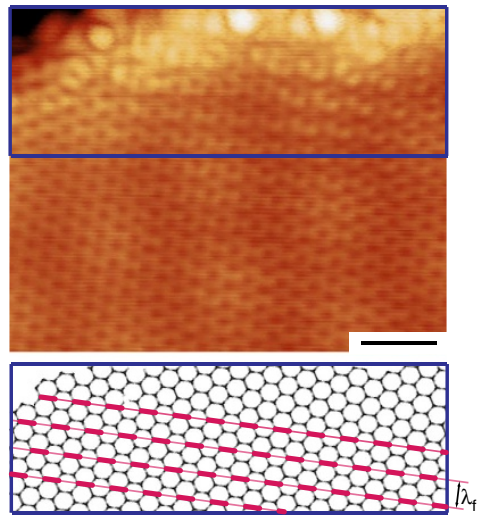

b

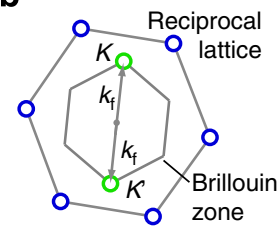

c

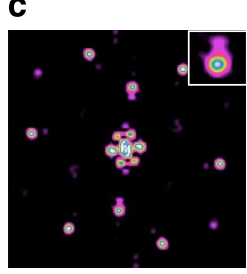

d

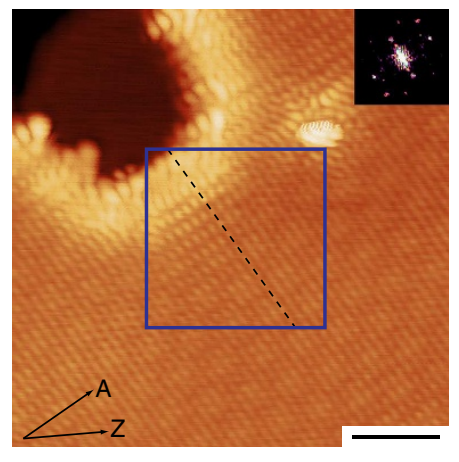

e
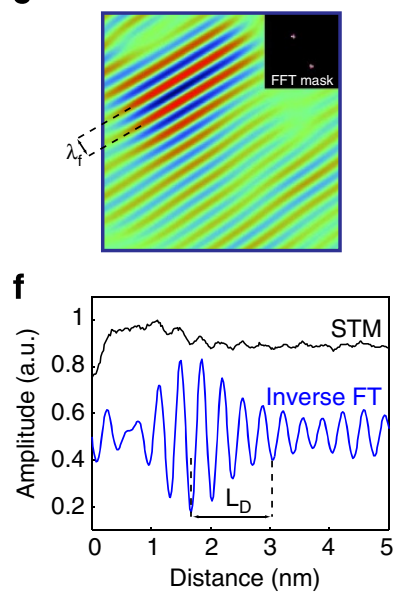

Figure 5 | Electronic scattering at graphene edges. (a) STM image of electronic scattering from a graphene edge (scale bar, $1 \mathrm{~nm}, V=25 \mathrm{mV}, I=15 \mathrm{nA}$ ). Hexagonal lattice below gives suggested edge termination based on atomically resolved image; light pink lines indicate periodicity of the electronic interference with the thicker pink lines highlighting the C-C bonds where the charge is localized. (b) k-Space map of the 2D Fourier transform (FT) (c) of the image in $\mathbf{a}$, showing the reciprocal lattice and $K / K^{\prime}$ points. (d) Electronic backscattering from an armchair edge in the growth front with the lattice directions given in the lower left hand corner: A, armchair and Z, zig-zag (scale bar, $2 \mathrm{~nm}, V=25 \mathrm{mV}, I=15 \mathrm{nA}$ ). Inset: 2D FT showing the RL and $K / K^{\prime}$ points. (e) Inverse FT image of the boxed region from $\mathbf{d}$ to highlight the spatial dependence of the $k_{f}\left(\lambda_{f}\right)$ scattering with the FT mask shown in the inset. (f) Height profile along the dashed line indicated in $\mathbf{d}$ and corresponding profile of the inverse FT in $\mathbf{e}$.

a higher atomic $\mathrm{C}$ concentration. This result reaffirms the observation that a gradient in both carbon dose and graphene coverage exists across the sample. The O1s spectra also demonstrate noticeable dissimilarities, most obviously the large tail in the curve taken in regions ii-v. The asymmetry in Fig. $3 \mathrm{c}$ is explained by an increased ratio between the two peaks at 531.8 and $530.5 \mathrm{eV}$, which are commonly attributed to carboxyls and the electrophyllic oxygen state on Ag, respectively ${ }^{39}$. The strong increase in the peak at $531.8 \mathrm{eV}$ in regions $\mathbf{i i - v}$ is attributed to the large portion of graphene edge states that interact with the oxidized/tarnished Ag surface after the sample is exposed to atmosphere. Finally, the decrease in the $530.5 \mathrm{eV}$ peak from region $\mathbf{i}$ to regions $\mathbf{i i - v}$ suggests a decrease in $\mathrm{Ag}-\mathrm{O}$ species that indicates that the graphene is protecting Ag from oxidation.

Electronic characterization. The electronic structure of the dendritic graphene was collected simultaneously with the topography via STS at energies of -200 and $-60 \mathrm{meV}$, and is overlaid on a 3D rendering of the topography in Fig. 4a,b, respectively. Electronic scattering at the Ag surface state is visible as standing waves on the terraces in both images, clearly differentiating the Ag from graphene. At both energies, the graphene exhibits a distinct electronic structure near its edges: an enhanced electronic density of states (DOS) at $-200 \mathrm{meV}$ and a depressed DOS at $-60 \mathrm{meV}$. The 3D topography illustrates that the differences in the $d I / d V$ mapping are predominantly electronic in nature-dissimilar from the topographically raised edges observed in GNRs ${ }^{40,41}$. The edge state at $-200 \mathrm{meV}$ has a decay length of $\sim 1.5-2 \mathrm{~nm}$ into the bulk graphene and generally exhibits a $30-50 \%$ DOS enhancement. Furthermore, the edge state observed in Fig. 3 is not only confined to the perimeter of the graphene flakes, but can be observed at grain boundaries in the graphene as well (highlighted in Fig. 4a). Supplementary Fig. S3 shows the dispersion of the edge state from -1 to $1 \mathrm{~V}$. The origin of the edge states observed here remains under investigation, but is currently thought to be unique to the $\mathrm{Ag}$-graphene system, as they are not seen in the $\mathrm{Cu}$-graphene system (under similar growth conditions).
The $d I / d V$ point spectra of the graphene and bare $\operatorname{Ag}(111)$ are shown in Fig. 4c. Notably, the surface state in the Ag spectrum appears near the Fermi level $\left(E_{\mathrm{f}}\right)$; however, the Dirac point (DP) is not clearly visible in the graphene spectrum. Theoretical work has predicted electron transfer from Ag to graphene under equilibrium separation, which would set the DP at between -200 and $-300 \mathrm{mV}$, similar to what is observed on $\mathrm{Cu}$. Although the DP is not readily apparent, light doping is consistent with the slightly blue-shifted G-band in the Raman spectrum at $\sim 1,589 \mathrm{~cm}^{-1}$. In addition, the fact that the two materials differ significantly across the entire energy range provides strong evidence that the graphene is weakly coupled to the underlying substrate. In comparison, the electronic spectrum of graphene on $\mathrm{Cu}(111)$ is nearly identical to the clean $\mathrm{Cu}(111)$ spectrum ${ }^{42}$, with only a slight suppression of the $\mathrm{Cu}$ surface state. The electronic contrast between the two metal systems suggests a weaker binding of graphene on Ag than $\mathrm{Cu}$, which is already considered to be in the weak binding regime $e^{43}$. Furthermore, the observation of several Moiré patterns indicates that the graphene is not in registry with the Ag lattice.

\section{Discussion}

Carrier scattering from graphene defects and edges has been predominantly studied on insulating substrates with the understanding that they are less perturbative to the electronic character of the graphene ${ }^{18,19}$. Previous work has established that intervalley backscattering is allowed only at armchair edges in graphene because of the limitations placed on the crystal momentum at the edges of the graphene ${ }^{37}$. This backscattering has been imaged via STM as a standing wave pattern at the edge of the graphene with a wavelength of $\lambda_{\mathrm{f}}=2 \pi / k_{\mathrm{f}}=3 \mathrm{a} / 2$ on nonmetallic substrates ${ }^{18,19}$ and $\lambda_{\mathrm{f}} / 2$ on a CVD copper foil ${ }^{44}$, where $k_{\mathrm{f}}$ is the Fermi wavevector and $\lambda_{\mathrm{f}}$ is the associated wavelength. The observed difference has been attributed to electronic confinement of the scattered waves to $\mathrm{C}-\mathrm{C}$ bonds in the graphene on the insulators, whereas the confinement is absent on $\mathrm{Cu}$ because of the coupling between the graphene and $\mathrm{Cu}$ surface ${ }^{18,44}$.

Figure 5a illustrates a scattering pattern from a graphene edge on $\mathrm{Ag}$, with the proposed edge termination given below. Clearly 
shown in both the STM and Fourier transform (Fig. 5b,c), the observed scattering has a wavelength (wavevector) of $\lambda_{\mathrm{f}}\left(k_{\mathrm{f}}\right)$. Data collected from $\sim 20$ atomically resolved images yields $\lambda_{\mathrm{f}}=$ $3.62 \pm 0.13 \AA$, which is in good agreement with the theoretical prediction of $\lambda_{\mathrm{f}}=3 \mathrm{a} / 2=3.69 \AA$. This $\lambda_{\mathrm{f}}$ quantum interference (Fig. 5d) also characteristically decays into the bulk graphene, consistent with previous observations ${ }^{19}$. A line profile (Fig. 5f) of the inverse Fourier transform shown in Fig. 5e displays a decaying profile away from the armchair edge with a decay length of $\mathrm{L}_{\mathrm{D}}=0.86 \pm 0.17 \mathrm{~nm}$. Finally, a splitting of the $k_{\mathrm{f}}$ scattering vector is shown in the inset to Fig. $5 \mathrm{c}$, similar to the work on $\mathrm{SiC}$, indicating the presence of long-range oscillations in the scattering envelope ${ }^{18}$. The presence of standing waves at graphene edges with periodicity $\lambda_{\mathrm{f}}$ indicates that the DOS can be localized along the $\mathrm{C}-\mathrm{C}$ bonds as on insulating substrates.

In conclusion, we report the successful growth of monolayer graphene on single-crystal $\mathrm{Ag}(111)$ under UHV conditions, using evaporated atomic carbon. The growth was conducted at relatively low substrate temperatures, making it a promising method for other low-melting-point substrates. The graphene growth was shown to emanate from small nanoparticles both at atomic steps and on terraces, generally leading to dendritic growth with large proportions of edges. The observation of quantum interference at the graphene edges highlights the weak interaction between the graphene and growth substrate. The ability to synthesize graphene on Ag in UHV opens the door to study interfaces with other $2 \mathrm{D}$ materials such as silicene.

\section{Methods \\ Graphene growth. The $\mathrm{Ag}(111)$ crystal was cleaned with multiple cycles of Ar ion sputtering $\left(1 \mathrm{kV}, 10^{-5} \mathrm{mbar}\right)$ at room temperature, followed by annealing in UHV at $550{ }^{\circ} \mathrm{C}$. Graphene growth was achieved through the deposition of elemental carbon onto a heated $\mathrm{Ag}(111)$ crystal at $\sim 700^{\circ} \mathrm{C}$. The carbon was evaporated from a $99.999 \%$ pure graphite rod using a UHV e-beam evaporator; the sharpened carbon rod required an $\sim 2 \mathrm{kV}$ accelerating voltage and $30 \mathrm{~mA}$ emission current to sustain a consistent flux. On the basis of coverages estimated from STM images (all taken with a tungsten tip at $55 \mathrm{~K}$ ), the deposition rate was well below $0.1 \mathrm{ML} \mathrm{min}^{-1}$, in agreement with the negligible pressure changes observed during deposition $\left(\sim 10^{-10} \mathrm{mbar}\right)$. Carbon was deposited for $20 \mathrm{~min}$, followed by immediate substrate cooling to room temperature.}

Scanning tunnelling microscopy. STM microscopy was performed using an Omicron VT System with a base pressure of $<10^{-12}$ mbar. The experiments were conducted with electrochemically etched tungsten tips at $\sim 55 \mathrm{~K}$. STS was simultaneously collected by applying a small periodic modulation to the applied voltage and collecting the current with a SR830 Lock-In Amplifier. XPS was conducted in a Thermo Scientific ESCALAB $250 \mathrm{Xi}$ at a base pressure of $10^{-9} \mathrm{mbar}$. Raman spectroscopy was taken with a Renishaw InVia Raman Microscope System with a 514-nm laser line.

\section{References}

1. Geim, A. K. \& Novoselov, K. S. The rise of graphene. Nat. Mater. 6, 183-191 (2007).

2. Geim, A. K. \& Grigorieva, A. Van der Waals heterostructures. Nature 499, 419-425 (2013).

3. Addou, R., Dahal, A. \& Batzill, M. Growth of a two-dimensional dielectric monolayer on quasi-freestanding graphene. Nat. Nanotechnol. 8, 41-45 (2012).

4. Sun, Z. et al. Towards hybrid superlattices in graphene. Nat. Commun. 2, 559 (2011).

5. Dean, C. R. et al. Boron nitride substrates for high-quality graphene electronics. Nat. Nanotechnol. 5, 722-726 (2010).

6. Grigorenko, A. N., Polini, M. \& Novoselov, K. S. Graphene plasmonics. Nat. Photon. 6, 749-758 (2012).

7. Liu, Y. et al. Plasmon resonance enhanced multicolour photodetection by graphene. Nat. Commun. 2, 579 (2011)

8. Lin, Y. -C. et al. Graphene annealing: how clean can it be? Nano Lett. 12, 414-419 (2012).

9. Liang, X. et al. Toward clean and crackless transfer of graphene. ACS Nano 5, 9144-9153 (2011).

10. Song, Y. J. et al. High-resolution tunnelling spectroscopy of a graphene quartet. Nature 467, 185-189 (2010).
11. Cao, Q. et al. Medium-scale carbon nanotube thin-film integrated circuits on flexible plastic substrates. Nature 454, 495-500 (2008).

12. Weiss, N. O. et al. Graphene: an emerging electronic material. Adv. Mater. 24, 5782-5825 (2012).

13. Vogt, P. et al. Silicene: compelling experimental evidence for graphenelike twodimensional silicon. Phys. Rev. Lett. 108, 155501 (2012).

14. Feng, B. et al. Evidence of silicene in honeycomb structures of silicon on $\mathrm{Ag}(111)$. Nano Lett. 12, 3507-3511 (2012).

15. Li, X. et al. Large-area synthesis of high-quality and uniform graphene films on copper foils. Science 324, 1312-1314 (2009).

16. Tsen, A. W. et al. Tailoring electrical transport across grain boundaries in polycrystalline graphene. Science 336, 1143-1146 (2012).

17. Iski, E. V., Yitamben, E. N., Gao, L. \& Guisinger, N. P. Graphene at the atomicscale: synthesis, characterization, and modification. Adv. Funct. Mater. 23, 2554-2564 (2013).

18. Yang, H. et al. Quantum interference channeling at graphene edges. Nano Lett. 10, 943-947 (2010).

19. Koepke, J. C. et al. Atomic-scale evidence for potential barriers and strong carrier scattering at graphene grain boundaries: a scanning tunneling microscopy study. ACS Nano 7, 75-86 (2013).

20. Li, X., Cai, W., Colombo, L. \& Ruoff, R. S. Evolution of graphene growth on Ni and $\mathrm{Cu}$ by carbon isotope labeling. Nano Lett. 9, 4268-4272 (2009).

21. Mattevi, C., Kim, H. \& Chhowalla, M. A review of chemical vapour deposition of graphene on copper. J. Mater. Chem. 21, 3324 (2011).

22. Karakaya, I. \& Thompson, W. T. The Ag - C (silver-carbon) system. J. Phase Equilib. 9, 226-227 (1988).

23. Zangwill, A. \& Vvedensky, D. D. Novel Growth mechanism of epitaxial graphene on metals. Nano Lett. 11, 2092-2095 (2011).

24. Wang, B., Ma, X., Caffio, M., Schaub, R. \& Li, W. -X. Size-selective carbon nanoclusters as precursors to the growth of epitaxial graphene. Nano Lett. 11, 424-430 (2011).

25. Starodub, E. et al. Graphene growth by metal etching on Ru (0001). Phys. Rev. B 80, 235422 (2009).

26. Nie, S. et al. Scanning tunneling microscopy study of graphene on $\mathrm{Au}(111)$ : Growth mechanisms and substrate interactions. Phys. Rev. B 85, 205406 (2012).

27. Giovannetti, G., Khomyakov, P. A., Brocks, G. \& Karpan, V. M. Doping graphene with metal contacts. Phys. Rev. Lett. 101, 026803 (2008).

28. Malard, L. M., Pimenta, M. A., Dresselhaus, G. \& Dresselhaus, M. S. Raman spectroscopy in graphene. Phys. Rep. 473, 51-87 (2009).

29. Wurstbauer, U. et al. Molecular beam growth of graphene nanocrystals on dielectric substrates. Carbon. 50, $4822-4829$ (2012).

30. Jerng, S. K. et al. Nanocrystalline graphite growth on sapphire by carbon molecular beam epitaxy. J. Phys. Chem. C 115, 4491-4494 (2011).

31. Garcia, J. M. et al. Graphene growth on h-BN by molecular beam epitaxy. Solid State Commun. 152, 975-978 (2012).

32. Ferrari, A. C. et al. Raman spectrum of graphene and graphene layers. Phys. Rev. Lett. 97, 187401 (2006).

33. Ferrari, A. C. Raman spectroscopy of graphene and graphite: Disorder, electron-phonon coupling, doping and nonadiabatic effects. Solid State Commun. 143, 47-57 (2007).

34. Bischoff, D. et al. Raman spectroscopy on etched graphene nanoribbons. J. Appl. Phys. 109, 073710 (2011).

35. Eckmann, A. et al. Probing the nature of defects in graphene by raman spectroscopy. Nano Lett. 12, 3925-3930 (2012).

36. Li, X. et al. Large-area graphene single crystals grown by low-pressure chemical vapor deposition of methane on copper. J. Am. Chem. Soc. 133, 2816-2819 (2011)

37. Cançado, L., Pimenta, M., Neves, B., Dantas, M. \& Jorio, A. Influence of the atomic structure on the Raman spectra of graphite edges. Phys. Rev. Lett. 93, 247401 (2004).

38. Rutter, G. M. et al. Scattering and interference in epitaxial graphene. Science 317, 219-222 (2007).

39. Demidov, D. V., Prosvirin, I. P., Sorokin, A. M. \& Bukhtiyarov, V. I. Model Ag/HOPG catalysts: preparation and STM/XPS study. Catal. Sci. Technol. 1, 1432 (2011).

40. Tao, C. et al. Spatially resolving edge states of chiral graphene nanoribbons Nat. Phys. 7, 616-620 (2011).

41. Pan, M. et al. Topographic and spectroscopic characterization of electronic edge states in CVD grown graphene nanoribbons. Nano Lett. 12, 1928-1933 (2012).

42. Gao, L., Guest, J. R. \& Guisinger, N. P. Epitaxial graphene on $\mathrm{Cu}(111)$. Nano Lett. 10, 3512-3516 (2010).

43. Batzill, M. The surface science of graphene: metal interfaces, CVD synthesis, nanoribbons, chemical modifications, and defects. Surf. Sci. Rep. 67, 83-115 (2012).

44. Tian, J., Cao, H., Wu, W., Yu, Q. \& Chen, Y. P. Direct imaging of graphene edges: atomic structure and electronic scattering. Nano Lett. 11, 3663-3668 (2011).

\section{Acknowledgements}

We thank M. Köppen and C. Linsmeier for their help with the carbon e-beam evaporation. Use of the Center for Nanoscale Materials was supported by the U.S. Department of 
Energy, Office of Science, Office of Basic Energy Sciences, under Contract No. DE-AC0206CH11357. B.K. acknowledges support from a National Science Foundation Graduate Research Fellowship (DGE-0824162). This work was also supported by the US Department of Energy SISGR contract number DE-FG02-09ER16109.

\section{Author contributions}

B.K., E.V.I. and N.P.G. performed the graphene growth, STM and STS characterization. B.K. and A.J.M. performed the XPS. B.K. performed the Raman spectroscopy. All authors contributed to discussion of the experiments. M.C.H. and N.P.G. guided the project. B.K. and E.V.I. wrote the manuscript with revisions from all authors.

\section{Additional information}

Supplementary Information accompanies this paper at http://www.nature.com/ naturecommunications

Competing financial interests: The authors declare no competing financial interests.

Reprints and permission information is available online at http://npg.nature.com/ reprintsandpermissions/

How to cite this article: Kiraly, B. et al. Solid-source growth and atomic-scale characterization of graphene on $\mathrm{Ag}(111)$. Nat. Commun. 4:2804 doi: $10.1038 /$ ncomms3804 (2013). 